\title{
ANALYSIS of Control Force Grasping for a Multifunctional Five Fingered Robot to Pick-up Various of Components
}

\author{
W.Widhiada ${ }^{1}$, N.S.Kumara ${ }^{2}$ and T.G.T. Nindhia ${ }^{1}$ \\ ${ }^{1}$ University of Udayana, Mechanical Engineering Department, Campus Bukit Jimbaran Bali, Indonesia \\ ${ }^{2}$ University of Udayana, Electrical Engineering Department, Campus Bukit Jimbaran Bali, Indonesia
}

\begin{abstract}
Multi-fingered robot gripper has become popular in the major research topics as grasping an object in robotic systems. The author considers a matter of style-based control model for a multi-fingered robot hand grasping an object with a known geometric characteristics. This paper introduces design process and analysis of contact force the five fingered gripper suitable to handle several of objects. The author applied Simulink/SimMechanics, Support package Arduino and Inventor software packages to facilatate and integrated the design of contact force gripper systems. The advance of PID control is used to control dynamics motions of the five fingered gripper systems. The multifunction finger's gripper is developed to handle the various components. Contact force between fingertips and object surface is computed using the Hooke law concept. The analysis of experiment result shows the optimum of contact forces are achieved to hold the object. The spring and damper algorithm is used to compute the interaction of force between fingertips and object surface.
\end{abstract}

\section{Introduction}

During the last three decades, robotic hands have broadly been developed with the aim of copying the human hand in terms of dexterity and adaptive capabilities to function as either a manipulator or as a prosthetic device. Most robotic grippers have been specifically designed only to grasp on certain traditional forms of object. In this study the author has researched into the design and analysis of contact force a multi-functional fingered gripper.

The application of robotic gripper systems has become accepted practice for a variety of tasks including the automation of assembly. However, in the field of automatic assembly there are still several areas to be conquered, one such area is the assembly of relatively small mechanical elements which exhibit non rigid characteristics. For instance we human beings are blessed with tremendous dexterity and the human hand/brain can handle various material characteristics from very stiff materials to extremely compliant materials.

Robots gripper is designed to adapt with an environment in a human manner will often require the hands and such as robot grippers are referred to as humanoid. There are some research papers into human hand behaviour development [1], [2] and [3].

A robot gripper is the physical realisation of an electromechanical system to perform physical handling tasks automatically. A robot griper is an essential element of the robotic system and it is designed to suit industrial application to typically grasp, carry, manipulate and assemble the components. Proper gripper design can simplify the overall assembly, improving overall reliability system, and reduce implementation costs, and grippers have been widely used for automated manufacturing, assembly and packaging, etc.

Robot grippers are generally confronted with a situation in which a few contact points of each finger take place at the same time and must be preserved so that the captured object does not slip from the grasp. To this end, a number of methodologies to reach stable control and dexterous manipulation tasks have been suggested, and a number of related studies published [4], [5] and [6]. Including all these authors proposed a geometric approach to decide displacement and force control of this multi-loop mechanism.

The driver for this research study arose from the difficulty of gripper design process to be an actual griper robot which is used to assembly and manipulate some objects. In this research the authors use both a simulation and an experimental method. An integrated both MATLAB/Simulink and Autodesk-inventor are used to investigate the response of multifunctional of the five fingered robot gripper. This paper has focused upon investigating how to develop the modelling and controlling the contact force of five fingered gripper. The design and a prototype robot gripper five fingers are built in this research. The motion of dynamics robot gripper are controlled by an advance PID control to achieve the best performance include quickly response and high accuracy respectively.

The aim of this research is to develop the multifunction needs for the five fingered gripper to pick 
up and manipulate some objects. The approach of the spring damper algorithm model is used to compute the contact force between the gripper finger and an object.

This is novel in that the creation of a single gripper that can be precisely controlled the contact force to grasp a range of engineering components of widely different handling characteristics has not, to the author's knowledge, been previously studied. The technical challenge of the work is such that the research will focus on the application of simulation techniques and real prototype.

\section{Methodology}

\subsection{Research description}

The development and methods in robotics research have been always affected by the devices used. This is especially true when one considers the profound impact of recent technologies on robotics, especially the development of software modelling environments which have become indispensable when designing the complex systems like three fingered robot gripper.

Grasping tasks are confronted with multi faced at the varied geometrical and physical properties of work pieces. So, before work pieces are grasped that one need to look at the characteristics which define the work pieces type and the way of gripper is presumed to pick it up. The characteristics of workpiece which are of excellent importance for the grasping process.

This paper introduces the demonstration of gripper motion to pick-up several objects. Matlab/Simulink software package is applied to support the computation contact force gripper for each finger and understanding of the various dynamics systems and in particular how both the SimMechanics and support package Arduino toolbox are apllied to interface seamlessly with ordinary Simulink block diagrams to enable the mechanical elements and its associated control system.

The physical model of the five finger robot gripper is built using SimMechanics software and the application SimMechanics has also been verified with identical outputs when compared with Simulink simulations. These studies indicate that each finger of the gripper can be controlled using PID formulation. The author believes an interesting alternative for the gripper, besides its assembly task, is that by changing the trajectory plan and the force control demands the gripper can possibly be used to provide physiotherapy type motions. This paper also demonstrates the power of integrating the software environments of Autodesk-Inventor and SimMechanics to validate the concept of designed force control of a dexterous gripper.

SimMechanics is a toolbox especially development to design the robotic system with a suite of tools to specify bodies and their mass properties, their possible motions, kinematic constraints and coordinates system and to initiate and measure body motions.

In this paper the contact force results of the dynamics of a robotic gripper to pick-up several objects are presented. The design of five fingered robot gripper is drawn in Autodesk-Inventor software as follow the dimension of human finger. The dwaing of gripper design is transfered from phisical model in $3 \mathrm{D}$ to $\mathrm{xml}$ file into some block diagrams in Simulink/Simmecanics is shown in Figure 1. SimMechanics tool facilates a reserve to deriving equations and performing them with base blocks. The mechanical system should be break down into the building blocks into Simmechanics model.

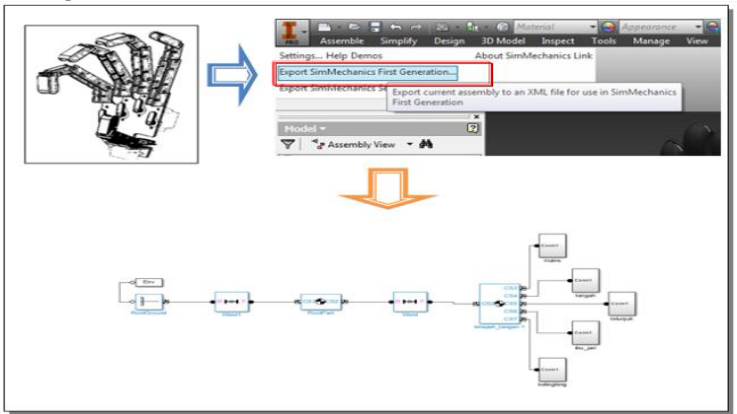

Figure 1. The process of design robot gripper.

The authors have designed the prototype of robot gripper to handle several objects. The contact force between finger tips and the surface of object are computed using the algorithm of force contact. In natural human grasping, the contact fingertip automatically adapts with the material strength. However, the hand struggles to pick up relatively small plane structures. The author has developed a mass spring damper model to determine the contact force response between the gripper finger and grasped object.

\subsection{Mathematics model for DC servo motor}

The robot gripper has used six DC servo motors. The mathematics model for DC servo motor is presented below.

$$
\frac{\theta(s)}{V_{a}(s)}=\frac{K_{t n}}{s\left[s^{2} J_{e f f} L+\left(L f_{e f f}+R J_{e f f}\right) s+R f_{e f f}+K_{t n} K_{b}\right]}
$$

where,

$\mathrm{R}=$ armature resistor, ohm

$\mathrm{L}=$ armature inductance, Henry

$\mathrm{Ktn}=$ constant of dc motor

$\mathrm{Kb}=$ constant of back voltage

$\mathrm{Va}(\mathrm{s})=$ applied armature voltage, volt

$\theta(s)=$ feedback voltage, volt

$\mathrm{J}_{\mathrm{eff}}=$ Moment inertia motor, $\mathrm{kg}-\mathrm{m}^{2}$

$\mathrm{f}_{\mathrm{eff}}=$ Coefficient viscous motor and load, N-/rad $/ \mathrm{s}$

The eq. 1 is the transfer function of DC servo motor which is used to drive the motor in each finger link Figure 2 shows the mathematics model of DC Motor is applied in block Simulink/Simmechanics.

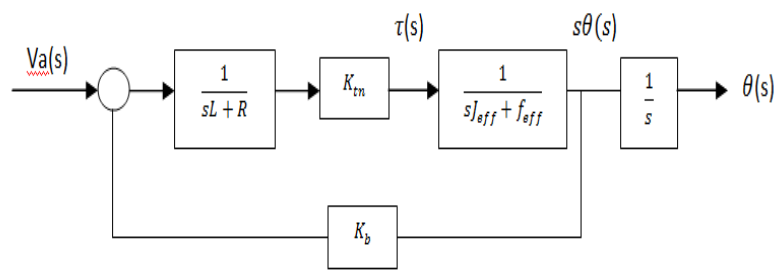

Figure 2. The transfer function block of DC servo motor. 


\subsection{Trajectory planning}

The input references are generated for the trajectory planning to the motion control system, which ensures that the five fingered gripper robot executes the planned trajectories.

Position and orientation of the object are also very important parameters where the position of the an object before the pick operation already sets limits to force induction options as the points where forces can be applied may not always be accessible. Grasping with a single contact surface is called adhesive grasp and this is the classic operating principle for suction grippers. On the contrary, form fit and force fit gripping are basic principles of grasping with more than one contact surface and the combinations of form fit and force fit gripping are frequently used. As described previously, the hand is great to any technical systems in terms of grasping flexibility as it naturally combines numerous grasping types.

\subsection{Gripper functionality}

Biagiotti proposed the basic concepts for human prosthetic which are the degree of anthropormorphism, the level of dexterity and controllability [7]. Anthropomorphism refers to the capability of prosthesis to the mimic the human hand, party or totally, considering some aspects such as shape, size, consistency, and other more general include colour, temperature, and so on. Dexterity is related to prosthesis functionality and not to shape or aesthetic factors. Generally speaking, with the term "dexterity" he intends the capability of the endeffector, operated by a suitable robotic system, to autonomously perform tasks with a certain level of complexity. An exhaustive review of scientific work done so far about robotic hands dexterity, with a complete list can be found in [8]. The dexterity attribute of prosthetic hands can be divided into two main performance indicates which are grasping and internal manipulation respectively. Grasping is the capability of holding objects inside the end-effectors volume under a given hand configuration. Internal manipulation is the controlled motion of the grasped object inside the hand workspace, with the hand constraining the object with a configuration variable during the manipulation [9].

The controllability is the responds to trend of designing prosthesis that easier to control by user, more than the design of more complex hands, having more degrees of freedom [10]. The controllability can be considered from several points of view such as from the point of view of patients and from the point of view of the controller.

Furthermore, another type of prosthetic hands develops to provide higher dexterity using anthropomorphical principles and improve the grasping function. The better performances of prosthetic hand are achieved by increasing the number of degrees of freedom and provide feedback signals such as position, velocity and grasping forces. Many papers are introduced in the past to mimic the extraordinary flexibility motion and use with a robotic gripper, which come close to the properties of a human hand, but clearly are lacking essential requirements for industrial use, such as robustness and cost [11].

In light of the review into published grippers and the experimental studies into how the range of the components should be grasped, the author believes that a minimum set of three actuated fingers are necessary to achieve the desired grasping functions. Philosophically the gripper is envisaged to mimic the thumb, index, middle, fore finger and a little finger. The gripper finger grasps a variety of tasks such as rigid bodies, and nonrigid bodies. However to cover all the gripping functions the fingers will require to additional functions. Thus the experimental of five fingered robot gripper has proposed to provide the desired grip to enable a variety of components to be correctly handled.

\subsection{Robust control strategy}

One of the fundamental requirements to grasp the object is the capability to handle interaction between gripper finger and the object. An interaction control method is needed to adapt the forces exerted on the object to avoid damage to both the object and the gripper fingers. Its aim is to control the dynamic behaviour of the gripper finger when contacting the object by controlling the properties of the contact, namely controlling the stiffness and the damping of the interaction.

The force control of robot hand is needed for ensuring that the hand firmly grasps and that corresponding finger in the presence of external forces applied to the object. These forces may require high values of contact force at the fingertip to get the stability response. To solve these problems Widhiada in 2015 published Robust Control For The Motion Five Fingered Robot Gripper adopted the relationship between the interaction force and position trajectory [12]. A considerable body of research has been undertaken on robotic hand system with robot force control and planning related to the entire device and the significant developments are as follows, position control is used when the robot finger must be moved with or without load along a prescribed trajectory through the robot finger workspace. The robust control system of robot fingers is simply a collection of joint controllers each of which is dedicated to a single joint and drives it individually. The reference signals for these controllers are supplied by a joint trajectory generator determining the desired joint trajectory from the desired trajectory of the gripper.

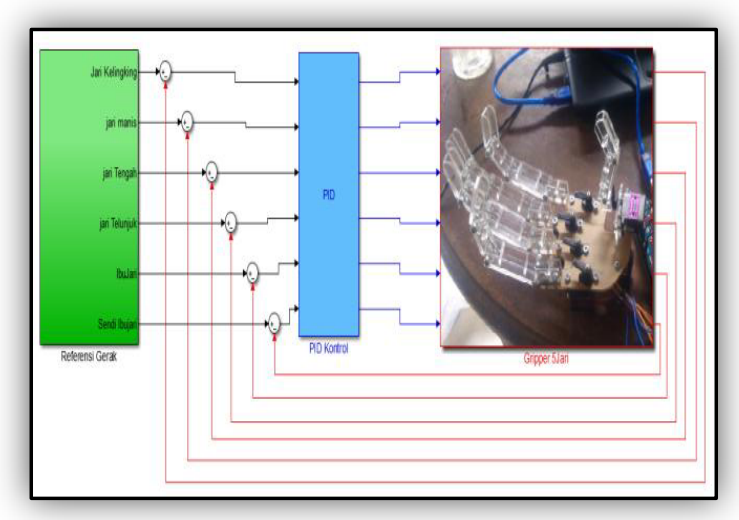

Figure 3. Robot gripper control system. 
The advance PID control has been used to control the dinamics motion of each of the fingers surface. By tuning suitable values of the three constant gains in the PID controller algorithm such as gain of Proportional (Kp), Integral (Ki) and Differential gain $(\mathrm{Kd})$ value are determined by auto tuning to achieve a quick response to get the steady state small eror signal. Parameter Kd works on the change of the error signal, so it can reduce dramatically overshoot effects to reach steady state response. Figure 3 shows the basic concept of Force control the Five fingered gripper using PID control.

\section{Result and Discussion}

Block diagram for computer interface is made in MATLAB/Simulink integrated with support package Arduino Uno. The advance PID control with auto tune is used to control the motions and force of five fingered gripper.

The value of force is used to drive the joint of servo motor can be compuetd from the angular position of joint servo motor. Figure 4 shows a link of gripper finger wich is installed at shaft servo motor.

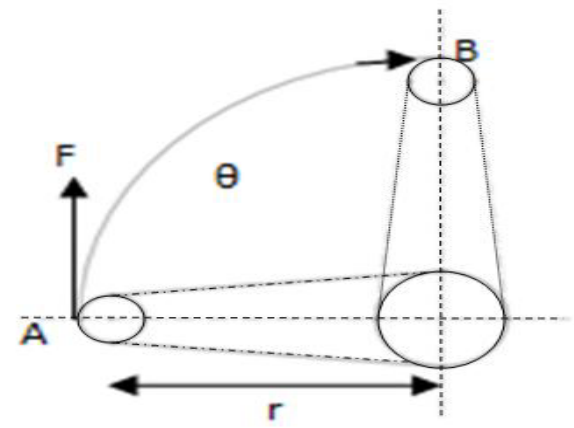

Figure 4. The lever of DC servo motor.

The force can be calculated when the tip of the motor lever moves from point $\mathrm{A}$ to $\mathrm{B}$ with the angle $\theta$ and the length of the lever of $r$. The first step is to calculate the force $(F)$ which makes the angle of displacement in the form of rad. Calculation results of the force on each lever the motor is shown in the Figure 5.

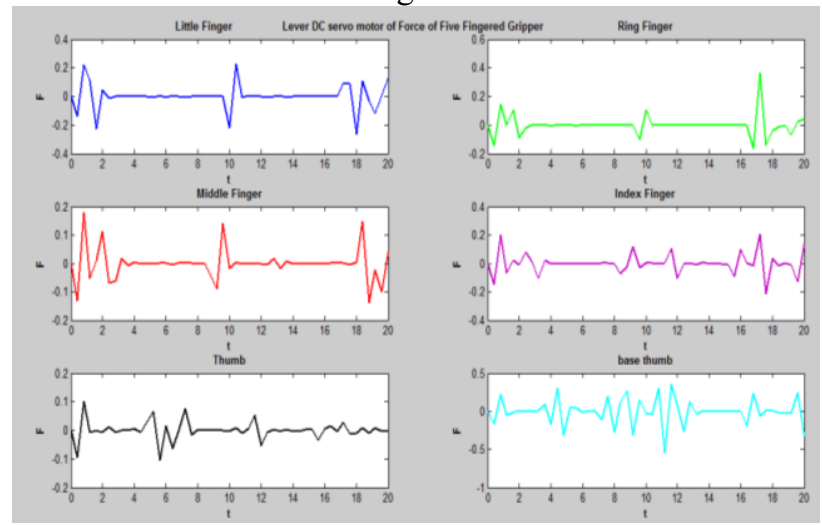

Figure 5. Contact force of servo motor.

The force $(\mathrm{F})$ is a positive value means of the motor lever moves from position $0^{\circ}$ to position the angle formed by reference. Conversely, if a force $(\mathrm{F})$ is negative, then the motor lever moves towards $0^{\circ}$.
The contact force of the gripper fingers can be calculated. Spring mounted on the tip of the finger will press the surface of the object. By using Hooke law the contact force between fingertips and an object surface can be computed by the equation 2 .

$$
F=-k \cdot \Delta x
$$

Figures 6-8 show the gripper grasp an object The contact force can be computed using a mass sprng damper model algorithm. In this test, the gripper will be instructed to hold some objects. The first objects to be held is a bottle. Dynamics motion gripper and the response of each lever of the dc servo motor on the gripper are shown in the graphs as follows.
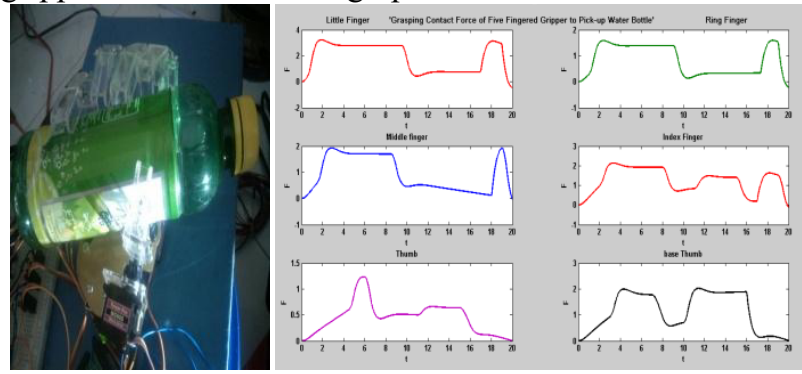

Figure 6. Bootle grasping contact force response finger

Figure 6 shows the gripper grasp a plastic bottle. Automatically the given contact force for the gripping the bottle is proportional to the weight of the bottle gripped. The maximum force occurs on the little finger is $3.22 \mathrm{~N}$, $2.32 \mathrm{~N}$ ring finger, middle finger $2.82 \mathrm{~N}, 3.21 \mathrm{~N}$ index finger, thumb $1.23 \mathrm{~N}$ and $2.01 \mathrm{~N}$ joint on the big toe. The average force acting on each finger when gripping namely: the little finger of $1.52 \mathrm{~N}$, the ring finger of $1,24 \mathrm{~N}, 1.43 \mathrm{~N}$ middle finger, index finger $1.54 \mathrm{~N}$, and $0.63 \mathrm{~N}$ thumb. Because the bottle tilted position when held resulted in one of the fingers have greater force than the other fingers.

In the second test, the gripper is tested for holding a disk or a small disk with a diameter of $900 \mathrm{~mm}$ in 20 seconds. The same condition when the gripper is used to grip a disk. The response shown in the graph shows that there are several fingers do not move reaches the reference angle. To force $(\mathrm{F})$ which occurs when holding a disk shown in the figure 7 .

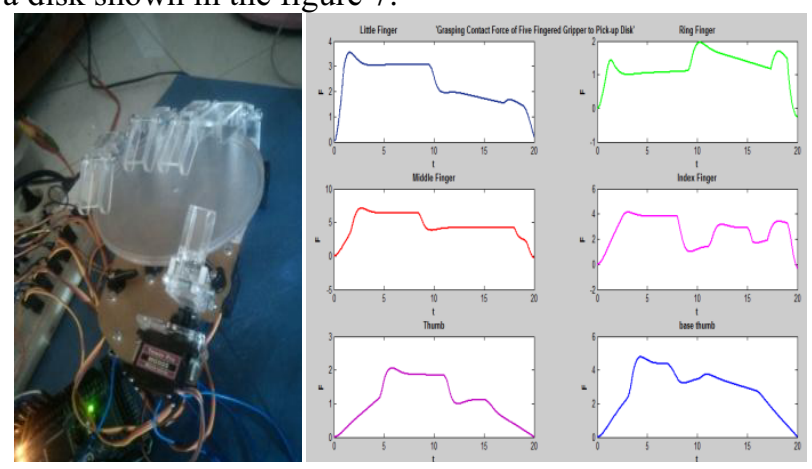

Figure 7. A disk grasping contact force response finger.

The magnitude of force given is proportional to the weight of disk gripped. The maximum force occurs on the little finger is $4.41 \mathrm{~N}, 2.25 \mathrm{~N}$ ring finger, middle finger $2.91 \mathrm{~N}, 2.75 \mathrm{~N}$ index finger, thumb $2.05 \mathrm{~N}$ and $2.86 \mathrm{~N}$ joint 
on the big toe. The average force acting on each finger when gripping namely: the little finger of $2.21 \mathrm{~N}$, the ring finger of $1.13 \mathrm{~N}, 1.82 \mathrm{~N}$ middle finger, index finger $1.43 \mathrm{~N}$, and $1.92 \mathrm{~N}$ thumb. Large diameter disk makes moving finger at a small angle so that the force $(F)$ which works also getting smaller.

Figure 8 shows the gripper is holding a pingpong ball. In the third test, a ping-pong ball will be used as an object to be grasped by a gripper in 20 seconds.
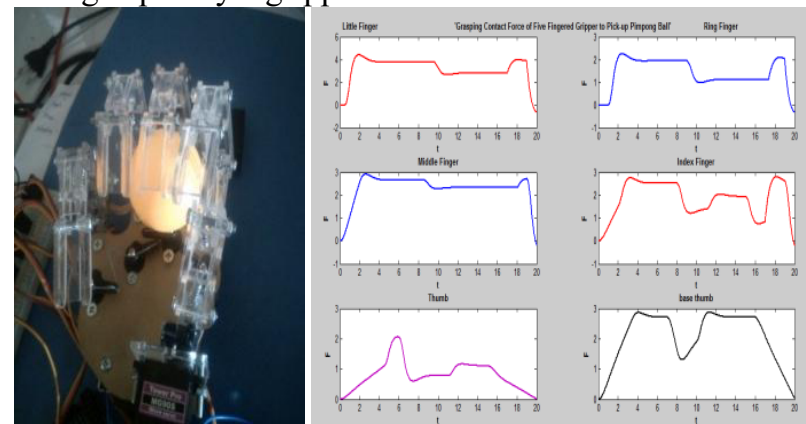

Figure 8. Grasping of a ping pong ball.

The magnitude of the force exerted gripper for holding a disk is proportional to the resistance of the object so that the disk is not damaged. The maximum force occurs on the little finger is $3.54 \mathrm{~N}, 1.96 \mathrm{~N}$ ring finger, middle finger $7.07 \mathrm{~N}, 4.11 \mathrm{~N}$ index finger, thumb $2.04 \mathrm{~N}$, and joints of the thumb $4.77 \mathrm{~N}$. The average force acting on each finger when gripping namely: the little finger of $1.45 \mathrm{~N}$, the ring finger of $1.98 \mathrm{~N}, 3.50 \mathrm{~N}$ middle finger, index finger $2.05 \mathrm{~N}$, and $2.40 \mathrm{~N}$ thumb. At the time of holding a ping pong ball, only three fingers are touching the surface of the ball is shown by the average of the force exerted is greater than the force of two fingers.

\section{Summary}

The dexterous design five finger gripper functionality is developed based on the philosophy of hand assembly. The creation of a single gripper can be precisely controlled to grasp a range variety of components. The developed of the advance PID controller to control the dynamics motion finger. This PID control provides the best performance for grasping of each component such as giving the smallest error, small overshoot, fast settling time and a stable system. The contact force is applied to determine the interaction forces between the fingertips and grasped object. The Newtonian law, using a spring damper is modelled for force reaction. The contact response force for each finger to holding object is different each other. The input references are provided only for grasping the object. When the finger touched the surface of object, the actual output response is smaller than the input reference. The contact force occurs when the finger is touching the surface of object by force displacement the lever of motor.

\section{References}

1. Fite, K.B., Withrow, T.J., Xiangrongshen, Wait, K.W. Mitchell, J.E. And Goldfarb, M., A Gas Actuated Anthropomorphic Prosthesis For Transhumeralamputees, IEEE Transactions On Robotics, Vol. 24, No. 1, 2008.

2. Kathryn, J., De Laurentis And Mavroidis,C., Mechanical Design Of A Shape Memory Alloy Actuated Prosthetic Hand, Ios Press, Technology And Health Care, Pp.91-106, 2002.

3. Massa, B. S. Et Al., Design and Development of an Underactuated Prosthetic Hand, In Robotics and Automation, Proceedings Icra/Ieee International Conference On, Vol. 4, Pp. 3374-3379, 2002.

4. Roberts, R.K., Paul And Hillberg, The Effect of Wrist Force Sensor Stiffness on the Control of Robot Manipulators, IEEE International Conference on Robotics, pp.269-274, 1985.

5. An, C.H. And Hollerbach, J.M.,Kinematic Stability Issue in Force Control of Manipulators, IEEE Int .Conf .onRobotics and Automation, Vol.4, pp.897 - 903, 1987.

6. Zhang, H., Kinematic Stability of Robot Manipulators under Force Control" IEEE International Conference on Robotics andAutomation, pp.80 - 85, 1989.

7. Biagotti, L., Melchiorri C. And Vassura G. , Control Of A Robotic Gripper For Grasping Objects In NoGravity Conditions" Proc. IEEE Int. Conf. Robotics And Automation, Seoul, Korea,Vol.2, Pp. 1427-1432, 2001.

8. Bicchi, A. And Kumar, V, Hands For Dexterous Manipulation And Robust Grasping, IEEE Transactions On Robotics And Automation, Vol.16, No.6, Pp.652-662, 2000.

9. Cutkosky, M.R. And P.K.Wright, On Grasp Choice, Grasp Models, And The Design Of Hands For Manufacturing Tasks, IEEE Transactions On Robotics And Automation, Vol. 5, No.3. Pp. 269, 1989.

10. Abboudi, R.L., A Biomimetic Controller For A Multifinger Prosthesis, IEEE Transactions On Rehabilitation Engineering,Vol.7, No. 2,Pp.121-129, 1999.

11. Lotti, F. And Vassura,G., A Novel Approach To Mechanical Design Of Articulated Fingers For Robots Hands, Proceedings Of The Ieee/Rsj Intl. Conference On Intelligent Robots And Systems, Switzerland, Pp.1687-1692, 2002.

12. W. Widhiada, T.G.T., Nindhia And N. Budiarsa, Robust Control For The Motion Five Fingered Robot Gripper, International Journal Of Mechanical Engineering And Robotics Research,Vol.4, No.3,Pp.226-232, 2015. 